\title{
A hybrid outdoor localization scheme with high-position accuracy and low-power consumption
}

\author{
Hongwei Du ${ }^{1,2^{*}}$, Chen Zhang ${ }^{1,2}$, Qiang $\mathrm{Ye}^{3^{*}}$, Wen Xu ${ }^{4}$, Patricia Lilian Kibenge ${ }^{3}$ and Kang Yao ${ }^{1,2}$
}

\begin{abstract}
As mobile devices, such as smart phones, become more and more popular, localization based on mobile devices has gained much attention in both the academia and industry. Although global positioning system (GPS) has been widely deployed in many fields, it has several disadvantages such as consuming too much power and requiring the line of sight to satellites. Cellular network-based localization is much more robust. However, this type of localization scheme cannot achieve enough accuracy for some localization-based services. With the existence of densely deployed WiFi access points, WiFi-based localization seems very promising in terms of positioning precision and power consumption. However, different than GPS and cellular network based localization, most of the existing WiFi-based localization schemes are supposed to be used in indoor environments. Note that indoor WiFi-based localization schemes cannot be simply ported to urban outdoor environments due to several factors such as weaker signal and more serious interference. In this paper, we present a hybrid outdoor localization scheme utilizing crowdsourced WiFi signal data and built-in sensors in smart phones, which can achieve high-positioning accuracy and low power consumption. Our experimental results show that the proposed hybrid localization scheme outperforms the widely-adopted GPSbased method in terms of both positioning accuracy and power consumption. In addition, the idea of crowdsourcing fingerprints is effective.
\end{abstract}

Keywords: Outdoor localization, Positioning accuracy, Power consumption

\section{Introduction}

Over the past decades, localization service has been applied to a variety of different applications. So far, the global position system (GPS) has been the dominant technology to enable outdoor localization. However, since GPS localization requires the line of sight to four or more satellites, it does not work well in certain urban regions, such as a downtown canyon surrounded by skyscrapers. Another problem with GPS is its high power consumption, which is a serious challenge to battery-based mobile devices. To tackle the problems with GPS, many researchers have proposed a series of alternative localization schemes, including cellular-based systems [1, 2],

\footnotetext{
*Correspondence: hongwei.du@ieee.org; qye@upei.ca

'Department of Computer Science and Technology, Harbin Institute of Technology Shenzhen Graduate School, Shenzhen, China

${ }^{2}$ Shenzhen Key Laboratory of Internet Information Collaboration, Shenzhen, China

Full list of author information is available at the end of the article
}

infrared-based systems [3], ultrasonic-based systems [4], and radio frequency (RF)-based systems [5-7]. In terms of whether distance estimation is required or not, these schemes can be divided into two groups: range-based and range-free methods. Range-based methods rely on the estimated distances to achieve localization while rangefree methods do not need the distance information.

In our research, we focus on the outdoor localization schemes that can be adopted by smart phones. Among the alternative schemes mentioned previously, RF-based systems, especially those based on WiFi [8], are very promising. WiFi fingerprinting localization [9] is a RFbased positioning scheme that has been well studied. The key information in WiFi fingerprinting localization is the received signal strength indicator (RSSI), which can be easily obtained by all WiFi-enabled devices. Since most smart phones are equipped with WiFi, WiFi fingerprinting localization is more feasible than most other localization methods. Technically, this schedule does not utilize the 
estimated distance information based on signal strength. Instead, it first collects the WiFi fingerprints of varied locations and thereafter use the collected fingerprints to find the position of mobile devices.

In this paper, we present a hybrid outdoor localization scheme for smart phones which is based on our previous work [10]. It exploits WiFi fingerprinting, sensor information, and GPS statistics. Technically, the proposed scheme utilizes two phases to locate mobile devices. In the first phase, an offline WiFi fingerprint database is constructed via corwdsourcing [11]. This database includes not only the WiFi fingerprint data but also the GPS statistics. In the second phase, the real-time WiFi and GPS measurements are used to match the records in the database in order to achieve localization. In order to improve the localization accuracy and matching speed, we devised a map tile method, which utilizes sensor readings to limit the matching space. Our experimental results indicates that the accuracy of the proposed localization scheme is higher to that of GPS. In addition, the proposed scheme outperforms GPS in terms of power consumption.

In summary, our contributions can be listed as follows:

1. We proposed a hybrid outdoor localization scheme, which takes advantage of WiFi fingerprints, sensor information, and GPS statics. The proposed localization scheme achieves both high positioning accuracy and low power consumption.

2. The training phase of WiFi fingerprinting localization is often impractical in real-life applications because too much work needs to be completed in this phase. The proposed scheme is based on crowdsourced data, which effectively eliminates the bottleneck of the training phase of WiFi fingerprinting localization.

3. We proposed a map tile mechanism, which utilizes sensor readings to limit the matching space. Specifically, with the map tile method, not all records in the constructed database needs to be retrieved to find the matching record, which improves the localization speed significantly.

4. A prototype localization system based on the proposed scheme is implemented in our research. Our experimental results indicates that the proposed localization scheme outperforms GPS in terms of both positioning accuracy and power efficiency in the real outdoor environments.

The rest of this paper is organized as follows. In Section 1, the related work on existing localization schemes is presented. The problem formulation and three important observations are included in Section 2. Section 3 describes the proposed hybrid localization scheme. Section 4 includes the details of our experimental results. Finally, our conclusions are presented in Section 5.

\section{Related work}

As mentioned previously, to tackle the problems with GPS, many researchers have proposed a variety of different schemes for outdoor localization based on mobile devices. In terms of whether distance estimation is required or not, these schemes can be divided into two groups: range-based and range-free methods.

\subsection{Range-based methods}

Range-based localization methods have been extensively studied in past decades. In [12-15], the authors built optimization models utilizing physical distance to solve localization problems. Generally, range-based methods are mainly based on relative distance, which can be obtained through measuring methods like time-of-arrival (ToA), time difference of arrival (TDoA), or propagation model generated from RSSI value.

Time of arrival (ToA): The ToA methods measure the distance from the unlocated devices to the anchor nodes through calculating the travel time of the signal. By doing so, the device can be located on a circle centered at the anchor node and the distance can be obtained by the time of arrival. Obviously, determining the precise position requires at least three anchor nodes and the estimated location is the intersection of these circles. However, due to the interference or accuracy, the circles may not intersect at the same point and therefore some filtering techniques, such as LS [16] or weighted least square (WLS) [17], are needed to estimate the position. In [18], the authors proposed an indoor localization named "Guoguo", which utilized the fine-grained adaptive time-of-arrival estimation approach.

Time difference of arrival (TDoA): By deploying the receivers at some known positions, the time of the signal arriving at each receiver are different, which can be exploited to measure the distance. The signal sender is on a hyperboloid of one receiver and different receivers have a constant range difference. This kind of methods use relative time measurements rather than absolute time measurements and time synchronization is only needed at the receivers. The estimated location is the intersection of the hyperbolic curves, and this kind of technique is also known as multilateration. Luo et al. [19] is an instance of TDoA. The authors utilized the time difference of arrival instead of the time of arrival as an distance indicator.

Propagation model: The received signal strength (RSS) could also be used for measuring distance. This type of methods are based on the path loss lognormal shadowing model. When a device detects available signals, it can calculate the distance between the base station and itself using the propagation model and the RSS. With the distance to at least three base stations, the location can be estimated using the trilateration. In [20], the authors proposed an indoor localization system called EZ, which 
consider the propagation of wireless signals as constraints and exploits it to locate the devices.

Angle of arrival (AoA): The angle of the arrived signals could also be used to locate the devices. When a device receives some signal from an anchor node, it can infer the region of the anchor node through the angle at which the signal is received. Typically, the region of the anchor node is a line. Therefore, at least two anchor nodes are required for this kind of localization methods. Besides, the error of the estimated position could be quite large since the estimation depends on both inferred region and the error can be scaled in the final result. Therefore, this kind of localization method is not implemented widely without large antenna arrays.

However, these range-based methods are sensitive to the surrounding environments and obstacles can easily produce errors. Other distance measuring technique like dead reckoning could assist user tracking, but produces accumulative errors over time.

\subsection{Range-free methods}

One of the most widely used range-free method is fingerprint localization method, especially in indoor localization. Basically, fingerprint methods in indoor localization can be categorized into three types: visual fingerprintbased localization, motion fingerprint-based systems, and signal fingerprint-based methods.

Visual fingerprint-based localization: With image processing ability, smart phones can acquire their positions through image matching methods. By taking a phone of one landmark, Google Goggles can identify it and then localize the device. In [21], the authors provides several corresponding visual-based localization systems. However, one of the greatest challenge in visual fingerprintbased localization is the matching speed. Additionally, the operation of camera also consume too much battery energy of smart phones. Furthermore, these kind of localization methods still cannot reach a high precision.

Motion fingerprint-based localization: Generally, to obtain the user's location, fingerprint-based localization methods need to detect the motion data of users, which can be obtained by the built-in sensors such as accelerator and gyroscope. CompAcc [22] finds out the moving pattern or trail of users to match with the pre-generated motion signatures. Unfortunately, since sensors may produce noise, with time goes on the errors generated by the sensors will accumulate.

Signal fingerprint-based localization: Signal fingerprint-based localization is widely used in places where a large number of WiFi infrastructures are deployed, especially in the indoor environment. Some outdoor applications such as [23] and [24] also use this method. These positioning methods commonly consist of offline training phase and online fingerprint matching phase. The goal of the first phase is to form a fingerprint database which stores the correlation between received signal strength(RSS) patterns from various access points(APs) and fix locations. Then the location of the device is determined at the matching stage. In this process we use matching algorithm to search the fingerprint in database which has the minimum difference with the device need to be located and the labeled location is our estimated location of user. Actually,fingerprints can be expressed in deterministic form (e.g., Radar [5]) and probabilistic form(e.g., Horus [25]). Calculating the difference between the patterns based on Euclidean distance are called deterministic form. And, probabilistic form can excellently store the information about the distribution signal strength at a fix position. However, using probabilistic method may generate time delay in mobile localization environment. This is because that we need to spend a lot of time in collecting enough samples to fit the fluctuating state of signals. In terms of effect, signal fingerprint-based localization has the ability to get fine-grained results. However, it is impractical to transfer this method directly from the indoor environment to the outdoor environment, because there are many problems such as complicated training work and complex outdoor environment.

\section{Preliminaries}

In this section, we present our problem formulation and three important observations related to WiFi fingerprinting and GPS localization. The notations used in this paper are listed in Table 1.

\subsection{Problem formulation}

In this paper,we use $f=\left\{r_{1}, r_{2}, \cdots, r_{n}\right\}$ to represent a fingerprint, where $r_{i}$ represents the RSS value of the AP

\section{Table 1 Table of notations}

\begin{tabular}{ll}
\hline Notation & Description \\
\hline$\hat{L}$ & The estimated location \\
$L_{i}$ & The location of fingerprint $i$ \\
$w_{i}$ & The weight of fingerprint $i$ \\
$P_{0}$ & The RSS value received at distance $d_{0}$ \\
$P_{d}$ & The RSS sensed in a distance of $d$ \\
$\gamma$ & The path loss exponent \\
$\rho_{i}$ & The discrimination factor of AP $i$ \\
$\rho_{i}^{N}$ & Normalized form of $\rho_{i}$ \\
$h\left(f, f^{\prime}\right)$ & The modified dissimilarity between $f$ and $f^{\prime}$ \\
$\sigma_{i}$ & The RSS strength value difference of AP $i$ \\
$\psi$ & The health of GPS \\
$s n r_{i}$ & Signal-to-noise ratio of satellite $i$ \\
$\eta$ & The ultimate synthesized dissimilarity metric \\
\hline
\end{tabular}


$A_{i} \in A$ and there are $n$ APs appear in $f$. We calculate the dissimilarity between two fingerprints based on RSS difference. For example, $\sigma_{i}=\left|r_{i}-r_{i}^{\prime}\right|$ is the difference of fingerprints $f^{\prime}$ and $f$ at each $A_{i}$. Due to the fact that two fingerprints may contain different set of APs, so it may appear that AP $A_{i}$ appears in $f$ but does not appear in $f^{\prime}$. For the above situation, we assume the signal strength is weak and let the missing value equal to -100 . The dissimilarity between $f^{\prime}$ and $f$ can be shown as

$$
\eta\left(f, f^{\prime}\right)=\sqrt{\sum_{i=1}^{p} \sigma_{i}^{2}}
$$

where $p=\left|A \cup A^{\prime}\right|$.

We found the sample with minimum dissimilarity through compare all samples stored in the fingerprint database $\mathcal{F}$ with the query fingerprint $f$.

$$
f^{*}=\underset{f_{i} \in \mathcal{F}}{\arg \min } \eta\left(f, f_{i}\right)
$$

$L\left(f^{*}\right)$ is the corresponding location of $f^{*}$ which is deliberated as the location of user. Locating errors is defined by $\epsilon=\left\|L(f)-L\left(f^{*}\right)\right\|$. As our problem is more complex, only considering the above content is inadequate. In the next section, we will present three important observations. Some improvements could be done in these three aspects and the proposed hybrid scheme has adopted these improvements.

\subsection{Three key observations}

WiFi fingerprinting and GPS localization work in different manners. Each of them has their advantages and disadvantages. In this section, we present three important observations based on our preliminary experiments about WiFi fingerprinting and GPS localization. These observations clearly indicate the key problems with these two schemes, which lead to either low positioning accuracy or high power consumption.

\subsubsection{High power consumption}

As we know, GPS is one of the most typical outdoor localization. It is an energy-hungry technology and two reasons lead to it's high battery energy cost. The first is that GPS antenna needs to continuously search for and communicate with the satellite at a low speed of data transmission. In this process, continued supply of electricity must be provided. The second is frequent network requests, which is happening when data is exchanged between user clients and the servers.

\subsubsection{Localization outliers}

The environment has a great impact on the accuracy of GSP positioning. In some interfering areas such as in forests or around buildings, the signals quality of GPS may be bad and get locating outliers. According to our preliminary experiments, we get the result shown in Fig. 1. It shows that GPS positioning abnormalities when the device bracket is near the building.

\subsubsection{Computational cost of global matching}

Most of previous research work use the global matching method to search the corresponding fingerprint information in the fingerprint database, which result in a lot of unnecessary computational consumption. Previous matching algorithms get the estimated location through searching the whole fingerprint database. But as the fingerprint database grows, computing costs and time delays are increasing. Although previous studies such as clustering method [25] have been proposed to reduce computational costs, updating fingerprints through crowdsourcing methods can still lead to additional computational overhead.

\section{A hybrid localization scheme for outdoor environments}

In this section, we present the details of the proposed hybrid localization scheme for outdoor environments. The major steps in the proposed scheme are included in Fig. 2. Technically, our localization scheme consists of three phases. The first phase is the traditional training phase. In this phase, the system will collect fingerprint data, including GPS status and corresponding WiFi signal state, through crowdsourcing technique and then build up a fingerprint database in server. In the second phase, the server will divide the map into map tiles using our map tile cache mechanism. The final phase is user online localization phase, where users' devices can calculate the dissimilarity between the collected patterns and the sample patterns in the database built before. The sample patterns are restricted in certain map tiles, which is stored in local cache and determined by sensor information, to reduce the computing time. And then the location of fingerprint with minimum difference will be selected as estimation for the location of the device. In the rest of this section, each phase will be presented in detail.

\subsection{Map tile mechanism}

As mentioned earlier, frequent network requests can generate very high energy cost and even have a long response time when the network is congested in typical positioning scheme. Although several offline applications have been proposed (e.g., big planet tracks [26]) to solve this problem, the effect of them is not ideal. So, in this paper we propose a cache mechanism based on map tiles, using geographic features to divide the map into many map tiles and maintain a local database cache. 


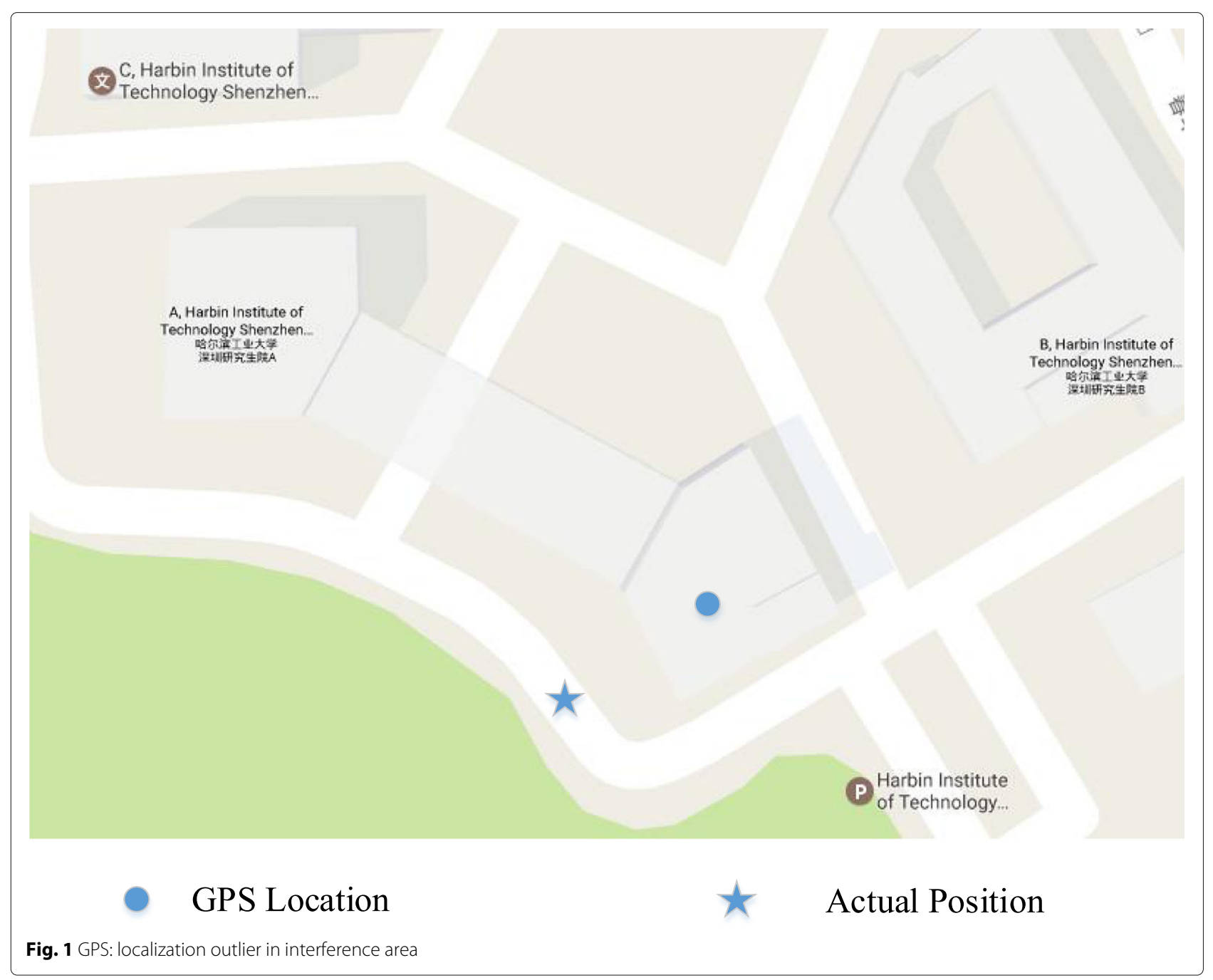

As Fig. 3 shows, we divide an area into $n$ map tiles and the length of each side is $w$ meters. According to the location (longitude and latitude) of uploaded fingerprint data, we allocate it into different map tile. And only $m$ map tiles is stored in client side local database. In Fig. 3, $m$ is equal to 4 . The premise of using the user device to sense the surrounding WiFi signal information is that we are able to ensure that the user is roughly located in the map tile in the local database through sensor assistance methods, which is described in detail in the next section. When the rough location is absent from the local fingerprint database, we need to update the cache from the server before proceeding with the prefetching operation, because the local data cache is out of date. When the total number of location requests is $r$, the typical fingerprint mechanism requires $r$ network requests, and our map tile cache mechanism requires only $r / m$ network requests. It can be seen that the proposed approach achieves the purposes of energy-saving and efficient.

\subsection{Sensor-assisted matching}

Comparing the measured RSS features fingerprint with the whole data base is inefficient and unnecessary. In this section, we introduce a sensor-assisted matching method which can restrict the matching operation in a small space through sensor information including direction and travel distance.

Figure 4 is an illustration. The current location can be calculated from the initial location $P$. Note that the initial location can be obtained by GPS or other localization methods. The distance and direction from the previous location to current location are denoted as $l$ and $\theta$, respectively. Actually, the distance and direction can be estimated using dead reckoning method with built-in inertial sensors like accelerometer, gyroscope, and compass. It indicates that the accuracy of count steps in dead reckoning can reach 98\% [27]. The moving distance $l$ can be obtained by multiply the user's step length and the footsteps. In [27], the authors also introduced how to 


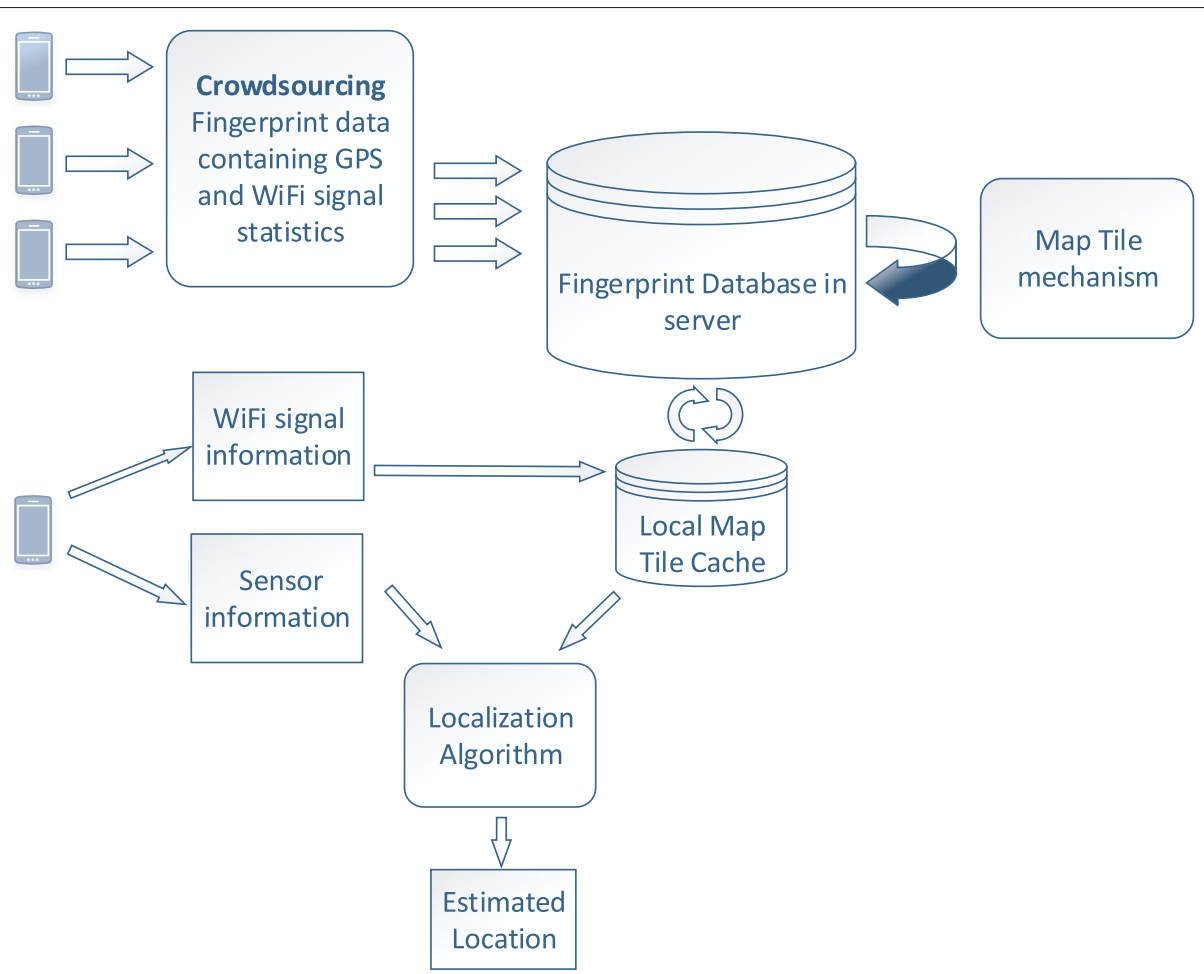

Fig. 2 System architecture

get direction $\theta$ using gyroscope and compass in detail. Because of the noisy sensors and heterogeneous devices, the distance $l$ and the direction $\theta$ cannot be precisely calculated. Two variables $\Delta l$ and $\Delta \theta$ are introduced here as fault tolerant range to cope with this problem. An annular region $A B C D$ can be obtained with all the above variables determined, which contains the current position. Estimating the current location requires finding out which fingerprint points in local cache are contained in this restricted area. Here, we offer a solution based on Haversine formula [28].
Given the longitude and latitude $(\varphi, \lambda)$ of the start point, and the distance $d$ and bearing $\theta$ from the start point, the destination point $\left(\varphi^{\prime}, \lambda^{\prime}\right)$ can be calculated using the following Eq. (3), where $\delta$ is the angular distance $1 / R$ and $R$ denotes the Earth's radius.

$$
\left\{\begin{array}{l}
\varphi^{\prime}=\arcsin (\sin \varphi \cdot \cos \delta+\cos \varphi \cdot \sin \delta \cdot \cos \theta) \\
\lambda^{\prime}=\arctan \left(\frac{\sin \theta \cdot \sin \delta \cdot \cos \varphi}{\cos \delta-\sin \varphi \cdot \sin \varphi^{\prime}}\right)+\lambda
\end{array}\right.
$$

Using the above formula, a subset of local fingerprint database cache can be obtained $\mathcal{F}=\left\{f_{1}, f_{2}, \cdots, f_{n}\right\}$.

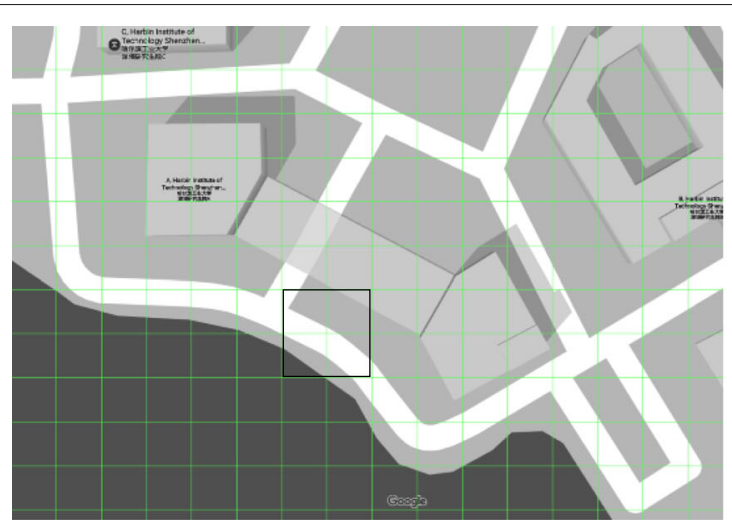

Map Tiles in Server
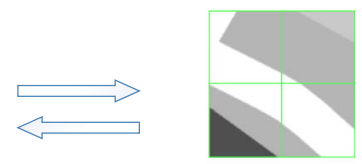

Map Tile Cache in Client

Fig. 3 Map tile mechanism 


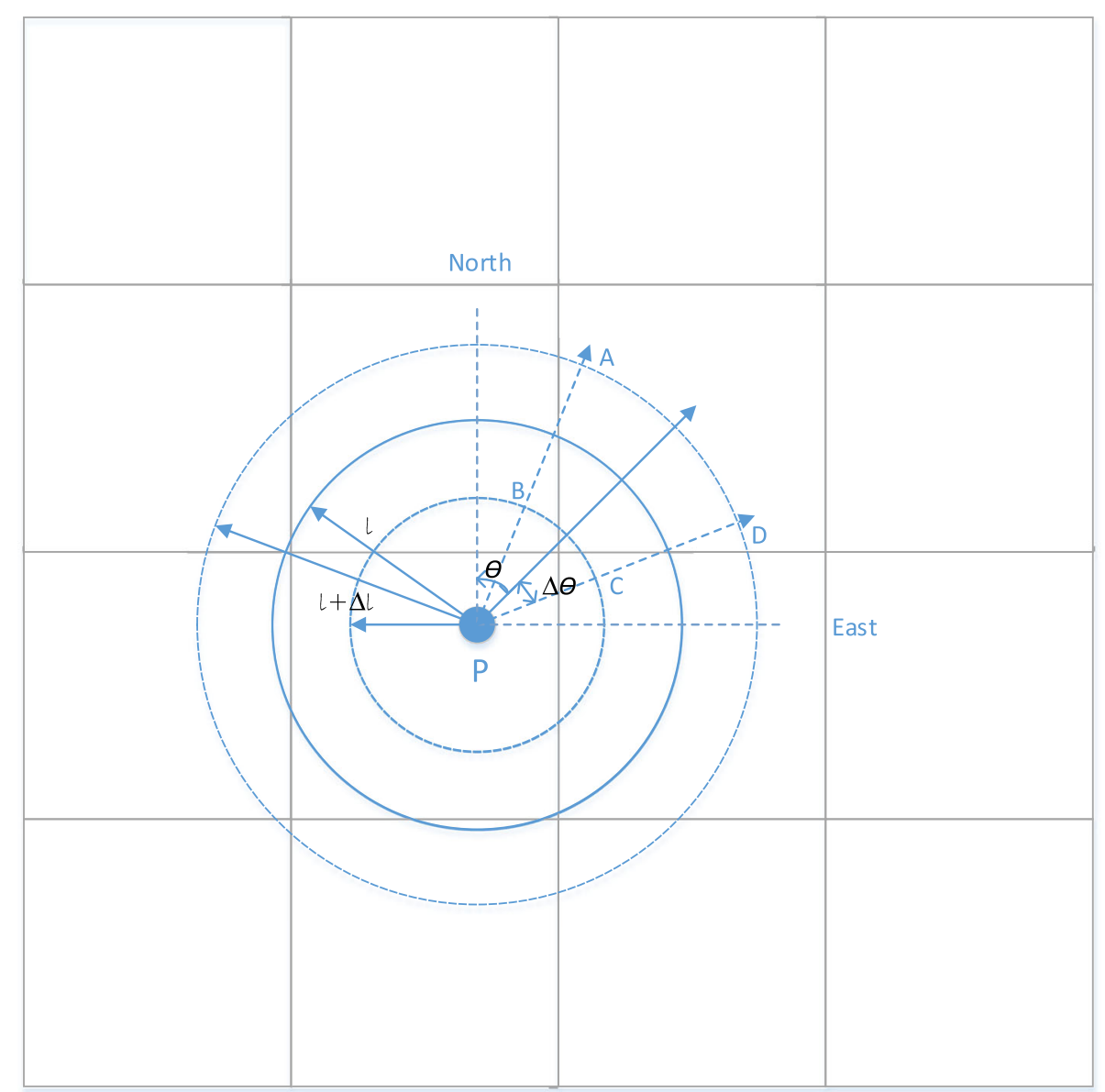

Fig. 4 Sensor-assisted matching in local map tile cache

Within this subset, the location $L_{i}\left(f_{i}\right)$ of every fingerprint $f_{i}$ locates in the constrained area $A B C D$.

\subsection{High-accuracy localization}

The observation in Section 3.2 shows that it costs much time for the probabilistic fingerprinting algorithm to collect samples for estimating current position. Therefore, we introduce a modified deterministic framework here. The basic idea is to estimate the result using weighted Knearest neighbor method. The weight of these fingerprints includes two aspects: the GPS signal state and dissimilarity in RSS. We will first introduce how to estimate the location $\hat{L}$ and then describe the calculation of weights.

The algorithm chooses each fingerprint sample $f \in \mathcal{F}$ to compare with query fingerprint signal $f^{\prime}$. Estimating the final result of current location $\hat{L}$ can be done with weighted K-nearest neighbor method [29] using the most similar $K$ points.

$$
\hat{L}=\sum_{i=1}^{K} \frac{w_{i}}{\sum_{j=1}^{K} w_{j}} L_{i}
$$

In the above equation, all weights $w_{i}$ are nonnegative and represents the dissimilarity in RSS and the stored GPS states like number of satellites $n$ and signal noise ratio $s n r$.

Different WiFi APs have different level of discrimination. Since the discrimination factor depends on the distance between AP and user device, by using Log-Distance Path Loss Model $P_{d}=P_{0}-10 \gamma \log \frac{d}{d 0}$, we specify the discrimination factor $\rho_{i}$ of the $i$-th AP with (5).

$$
\rho_{i}=\frac{1}{d_{i}}=10^{\frac{r_{i}-P_{0}}{10 \gamma}}
$$

In (5), $P_{0}$ is the RSS value received at distance $d_{0} \cdot \gamma$ is the pass loss exponent. And $P_{d}$ is the RSS sensed at distance $d$. Then, an normalized form $\rho_{i}^{N}$ from $\sum_{i=1}^{p} \rho_{i}$ can be used to calculate the modified dissimilarity in RSS between query fingerprint and stored one in (6).

$$
h\left(f, f^{\prime}\right)=\sqrt{\sum_{i=1}^{p}\left(\rho_{i}^{N} \cdot \sigma_{i}\right)^{2}}
$$

Moreover, the GPS satellite signal health is also an important part in ultimate synthesized discrimination. In 
our research, GPS satellite statistics is incorporated into synthesized influence factor. The GPS health here are represented by the number of effective satellites $n$ and corresponding signal-to noise ratio snr. The synthesized influence factor is expressed as follows:

$$
\psi=\frac{\sum_{j=1}^{n} s n r_{j}}{10 n}
$$

Note that with $\psi$ increases, the reliability of the stored location of this sample fingerprint increases.

Combine all these factors into unified one, the ultimate synthesized dissimilarity metric is formulated in (8), where $p=\left|A \cup A^{\prime}\right|$ and $q=\left|A \cap A^{\prime}\right|$. And $q$ denotes the number common APs in stored fingerprint $f$ and query one $f^{\prime}$. The dissimilarity of two fingerprints with fewer common APs will be amplified by $p / q$.

$$
\eta=\frac{h\left(f, f^{\prime}\right)}{\psi} \cdot \frac{p}{q}
$$

Finally, the absolute weight is obtained by (9), which can be used to calculate the current position in (4).

$$
w_{i}=\frac{1}{\eta}
$$

\section{Experimental results}

In this section, we first present our results on localization accuracy and power consumption, which were collected in a realistic testbed incorporating the prototype localization system. Then, we discuss the compatibility of crowdsourced RSSI data.

\subsection{Localization accuracy and power consumption}

In our research, we implemented a testbed around the Academic Building A at Harbin Institute of Technology Shenzhen Graduate School, China. In this testbed, a Samsung Galaxy Grand Prime running the proposed hybrid localization scheme was used to achieve localization. Before the experiment started, we divided the map area into many map square tiles and the length of them is

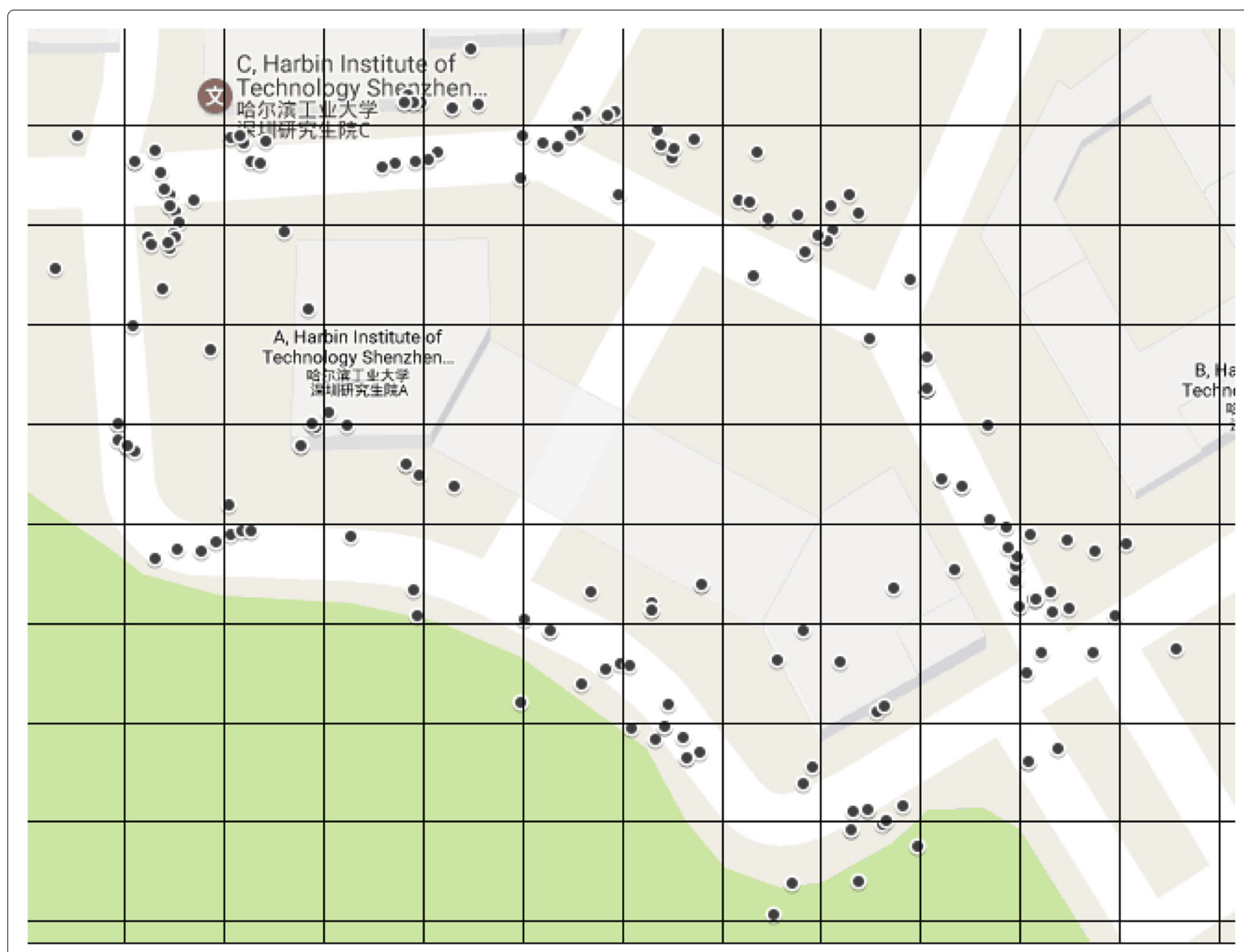

Fig. 5 Crowdsourcing data distribution in map tiles 
set to $w=15 \mathrm{~m}$. After that, we collected the fingerprint data at random locations along the way. The fingerprints are stored in a key-value format: the position and the corresponding series of RSS strength. Actually, we collected a total of about 150 sample points and the average state (including GPS statistics and signals information) of 10 samples is recorded as the fingerprint data. To eliminate the impact of the humans body on the experimental results, we unified the way of holding the device. After the training phase, we got a fingerprint database. And part of real fingerprint data in our database is allocated into corresponding map tile as the Fig. 5 shows.

According to the altitude and latitude of our location, we set the radius of the earth at ground level to about $6375.024 \mathrm{~km}$. The estimated position is calculated by Eq. (3) in online matching process. In fact, we selected 30 points along the road as the pre-calibrated point and repeat this process 10 times. In order to compare the mobile phone for GPS position and our hybrid method for the consumption of electricity, we recorded the battery energy percentage every ten minutes and form a power-consuming log.

According to the coordinates calculated by GPS, our hybrid localization and calibrated coordinates, we plotted these results into Google map as Fig. 6 shows. We compare the real position of 30 pre-calibrated points with GPS results and ours, respectively. The error cumulative distribution of GPS results and ours is shown in Fig. 7. It shows that the average error of our method results is $7.57 \mathrm{~m}$ while the number of GPS is 10.3. Figure 8 shows the error at each point. At point 17,21 and 24, our hybrid method gets much smaller errors than GPS. It proves that our method may handle exception values well to some extent. As Fig. 9 shows, our method increases the battery life by about 1.5 times. So, from the perspective of energy consumption, our approach meet the purpose of saving energy.

\subsection{Compatibility of crowdsourced fingerprints}

As mentioned previously, the first phase of the proposed localization scheme is the training phase, which is highly time-consuming because the data corresponding to each training point need to be measured and collected. For a small-scale system, although the training phase is not ideal in terms of time consumption, it is still feasible. However, for a large-scale localization system that covers a city or a province, the traditional training phase is infeasible in practice. This is why the proposed scheme attempts to utilize crowdsourcing to collect the required fingerprint data. Namely, if the training phase is completed by many incentive-based volunteers, instead of one individual, the training phase will take a much shorter period.

Despite the advantage of the crowdsourcing model, there is also a potential problem with this approach.

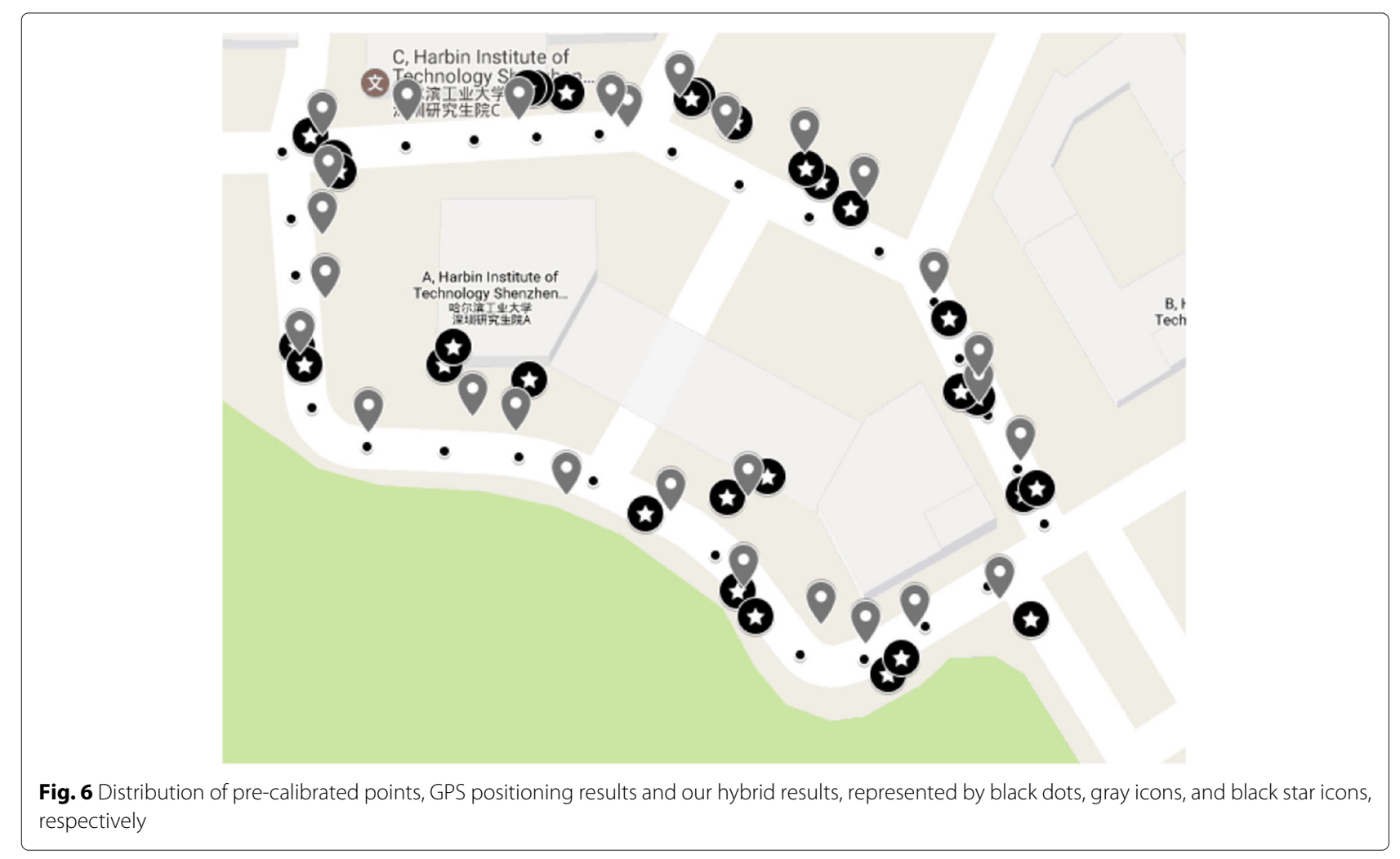




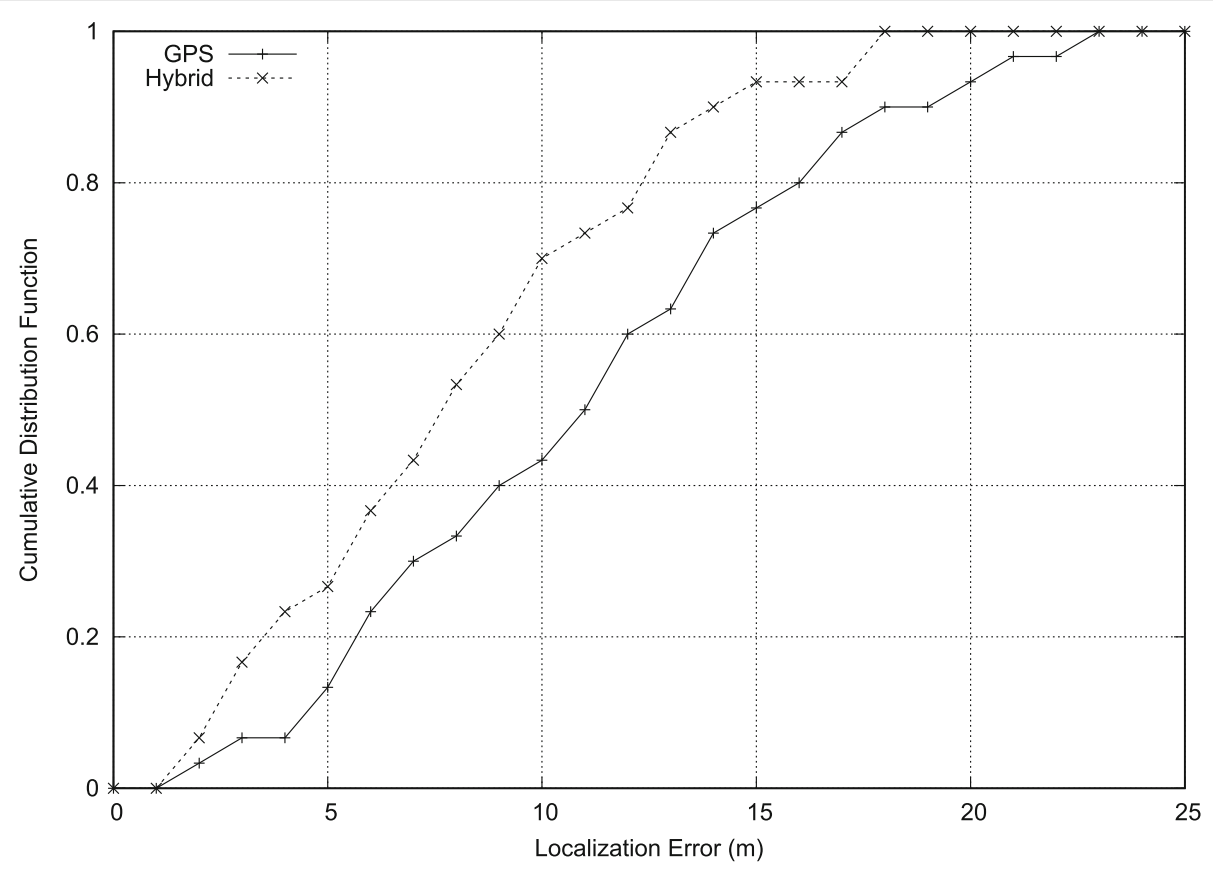

Fig. 7 Localization error: GPS and hybrid method

Namely, when fingerprints are collected by many volunteers and these volunteers might use all kinds of smartphone with varied reception capability, the collected fingerprints might not be compatible. That is, the fingerprints collected by two different smart phones at the same location might be highly different. If this takes place, the resulting localization accuracy will be degraded.

To verify whether the compatibility of crowdsourced fingerprints is a problem, we used two different Androidbased smart phones and three WiFi routers to conduct a series of experiments in a four-story building. Specifically,

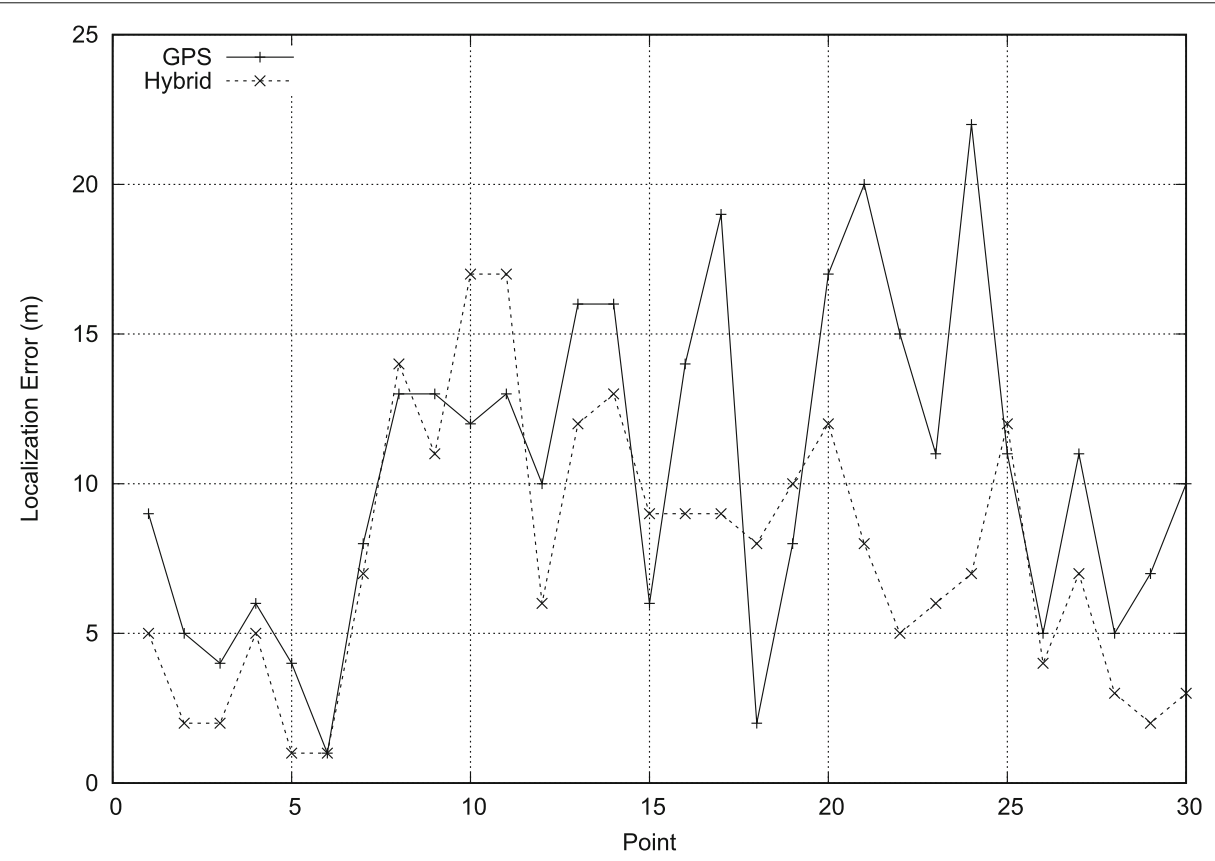

Fig. 8 Localization error at each point 


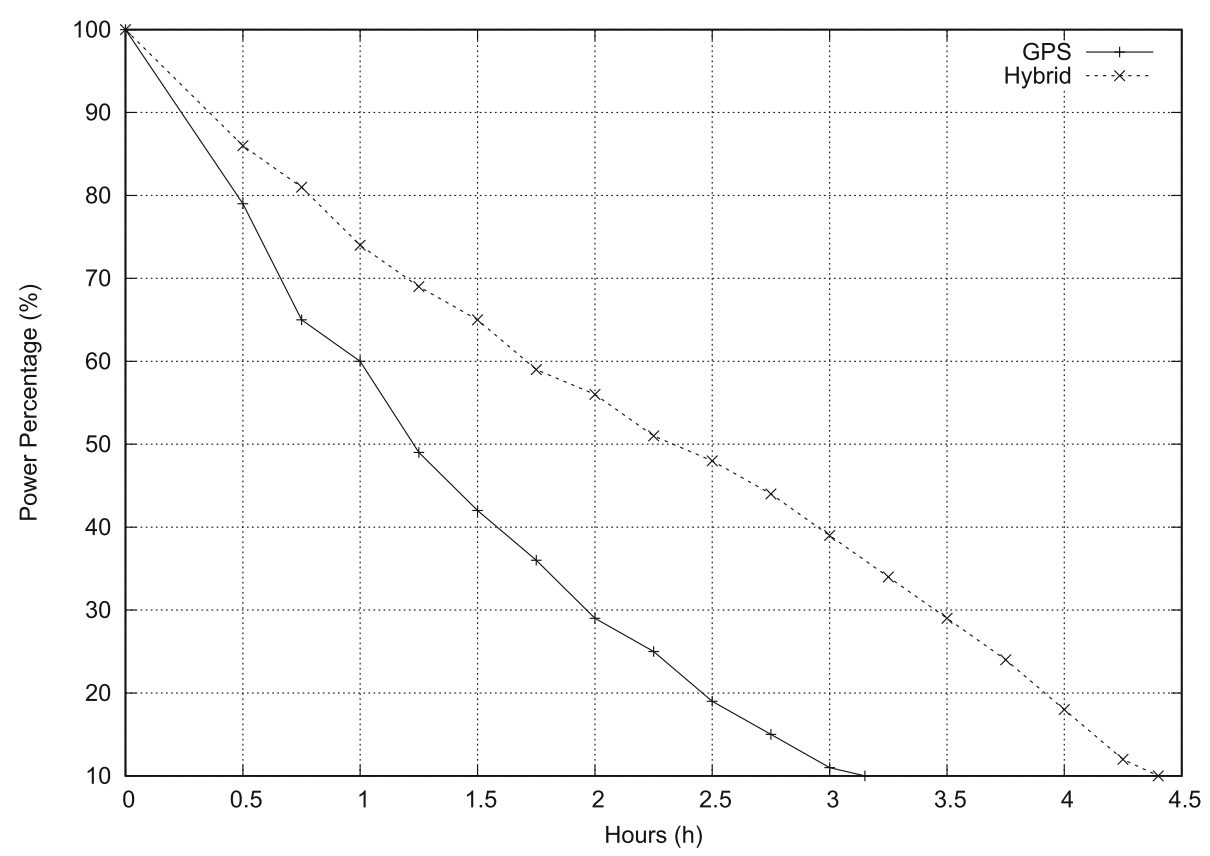

Fig. 9 Power consumption

the smart phones used in this set of experiments are Asus Zenfone 3 Laser and BLU Advance 5.0. The WiFi routers are D-Link DIR-819, D-Link WBR-2310, and Apple Airport Time Capsule. In our experiments, the two D-Link routers are placed on the third floor of the building and the Apple router is deployed on the second floor. In the first experiment, the smart phones are placed side-byside on the first floor. Then they start to collect the RSSI readings from the routers mentioned previously. After receiving 200 readings from each of the routers, the average of the 200 readings is used as the final RSSI value. In the second, third, and fourth experiments, the smart

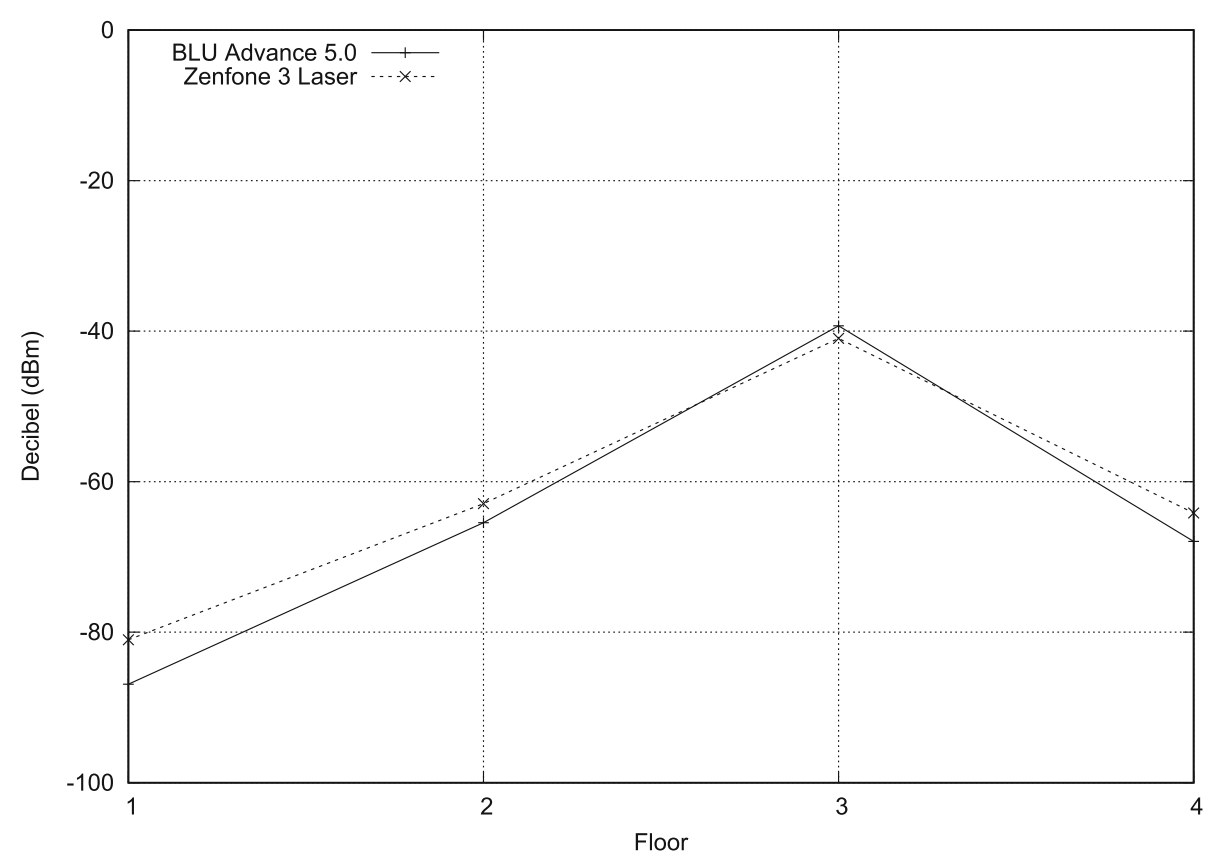

Fig. 10 RSSI comparison: D-Link DIR-819 


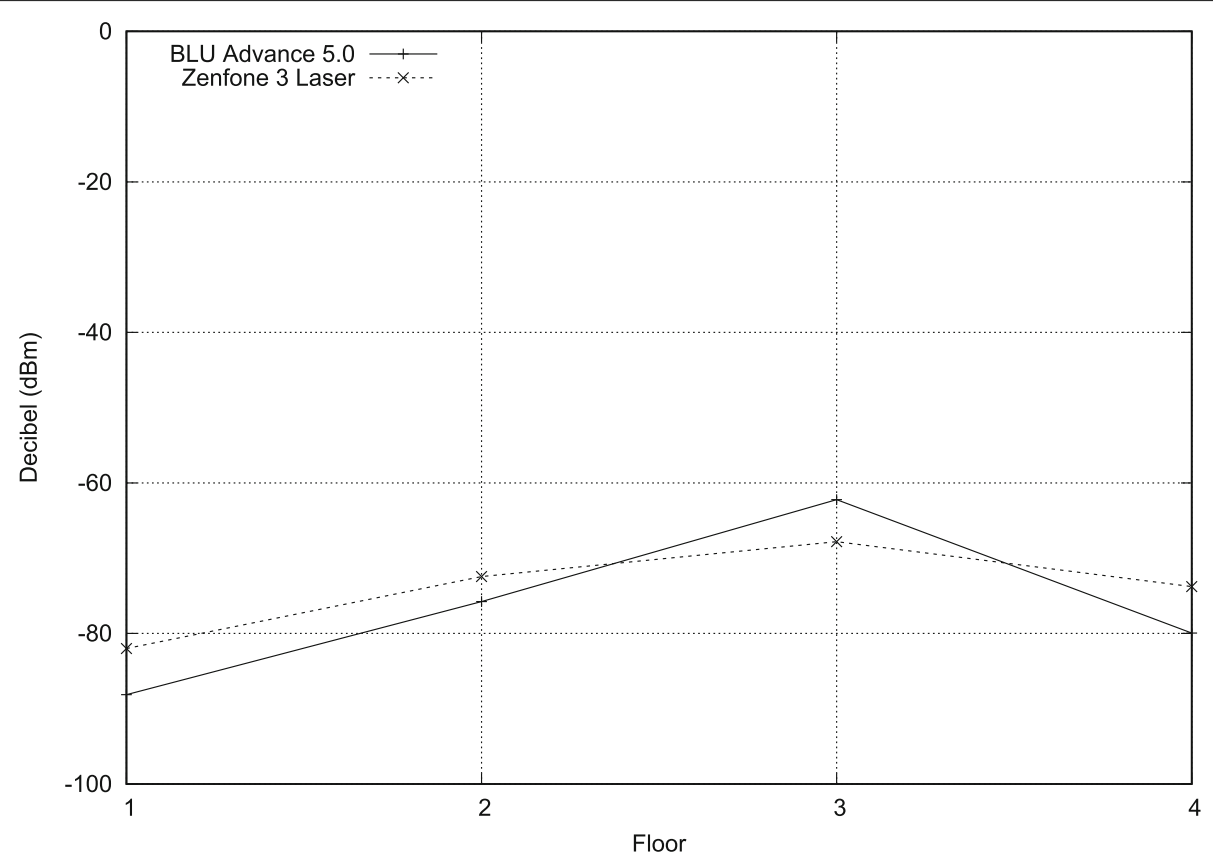

Fig. 11 RSSI comparison: D-Link WBR-2310

phones are deployed on the second, third, and fourth floor, respectively.

Our experiments results are summarized in Figs. 10, 11, and 12. In our research, we found that, overall, the compatibility of crowdsourced fingerprints is not a serious issue. Namely, the RSSI values collected by dif- ferent smart phones at the same location are very similar. Of course, the similarity varies with different routers. In our experiments, D-Link DIR-819 leads to the best compatibility. D-Link WBR-2310 an Apple Airport Time Capsule result in slightly degraded compatibility.

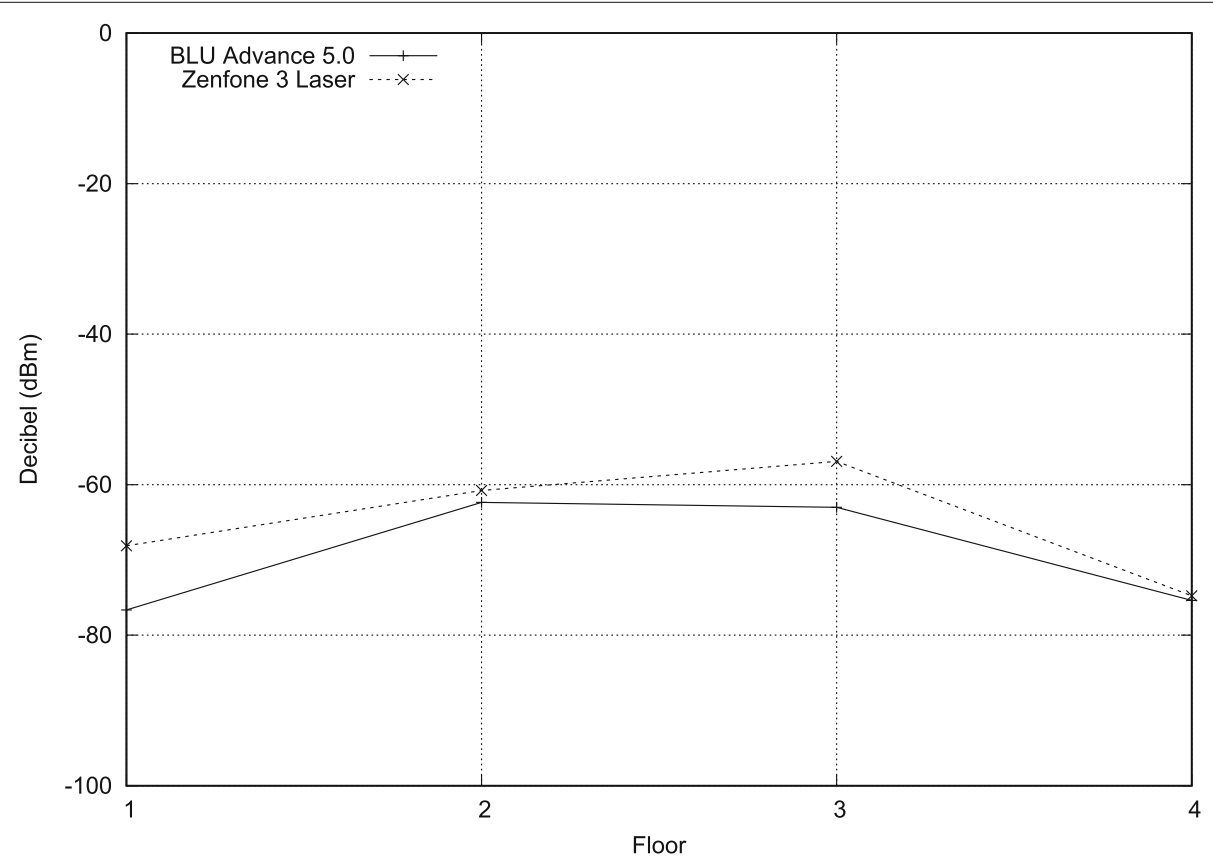

Fig. 12 RSSI comparison: airport time capsule 


\section{Conclusions}

In this paper, we present a hybrid outdoor localization scheme, which utilizes crowdsourced fingerprints and built-in sensors in smart phones. With the map tile mechanism and crowdsourcing feature, this scheme is expected to be more feasible than the existing outdoor localization methods. Our experimental results indicate that, compared with the widely-adopted GPS-based method, the proposed localization scheme leads to higher localization accuracy and lower power consumption. In addition, the idea of crowdsourcing fingerprints is effective.

\section{Acknowledgements}

Not applicable.

\section{Funding}

This work was jointly supported by National Natural Science Foundation of China (No. 61772154 and No. 61370216) and the Discovery Grant (Grant No.:RGPIN-2017-05853) from the Natural Science and Engineering Research Council, Canada.

\section{Availability of data and materials}

Not applicable.

\section{Competing interests}

The authors declare that they have no competing interests.

\section{Publisher's Note}

Springer Nature remains neutral with regard to jurisdictional claims in published maps and institutional affiliations.

\section{Author details \\ ${ }^{1}$ Department of Computer Science and Technology, Harbin Institute of Technology Shenzhen Graduate School, Shenzhen, China. ${ }^{2}$ Shenzhen Key Laboratory of Internet Information Collaboration, Shenzhen, China. ${ }^{3}$ School of of Mathematical and Computational Sciences, University of Prince Edward Island, Charlottetown, Canada. ${ }^{4}$ Department of Mathematics and Computer Science, Texas Woman's University, Denton, USA.}

Received: 7 September 2017 Accepted: 17 December 2017

Published online: 04 January 2018

\section{References}

1. M Ibrahim, M Youssef, in 2010 IEEE Global Telecommunications Conference, GLOBECOM 2010. Cellsense: A probabilistic rssi-based gsm positioning system (IEEE, Miami, 2010), pp. 1-5

2. M Ibrahim, M Youssef, Cellsense: An accurate energy-efficient gsm positioning system. IEEE Trans. Veh. Technol. 61(1), 286-296 (2012)

3. R Azuma, Tracking requirements for augmented reality. Commun. ACM 36(7), 50-51 (1993)

4. NB Priyantha, A Chakraborty, $\mathrm{H}$ Balakrishnan, in Proceedings of the 6 th Annual International Conference on Mobile Computing and Networking. The cricket location-support system (ACM, Boston, 2000), pp. 32-43

5. P Bahl, $\vee$ Padmanabhan, in Nineteenth Annual Joint Conference of the IEEE Computer and Communications Societies, INFOCOM 2000. Radar: an in-building rf-based user location and tracking system (IEEE, San Diego, 2000)

6. Y Wang, Q Ye, J Cheng, L Wang, in 201511 th International Conference on Mobile Ad-hoc and Sensor Networks (MSN). Rssi-based bluetooth indoor localization (IEEE, Shenzhen, 2015), pp. 165-171

7. MA Youssef, A Agrawala, AU Shankar, SH Noh, A probabilistic clustering-based indoor location determination system. Technical report (2002)

8. IEEE 802.11 Working Group, IEEE Standard for Wireless LAN Medium Access Control (MAC) and Physical Layer (PHY) Specifications. ANSI/IEEE Std. 802.11 (1999)
9. P Bahl, VN Padmanabhan, in Proceedings of the IEEE International Conference on ComputerCommunications (INFOCOM 2000). Radar: An in-buildingrf-based user location and tracking system (IEEE, San Diego, 2000), pp. 775-784

10. $\mathrm{K}$ Yao, H Du, Q Ye, W Xu, in 12th International Conference on Wireless Algorithms, Systems, and Applications, WASA 2017. A power-efficient scheme for outdoor localization (Springer, Guilin, 2017), pp. 534-545

11. J Howe, The rise of crowdsourcing. Wired. Mag. 14(6), 1-4 (2006)

12. Q Ye, J Cheng, H Du, X Jia, J Zhang, in Proceedings of the 15th ACM International Symposium on Mobile Ad Hoc Networking and Computing. A matrix-completion approach to mobile network localization (ACM, Philadelphia, 2014), pp. 327-336

13. J Cheng, Q Ye, H Du, C Liu, in 2015 IEEE 35th International Conference on Distributed Computing Systems, ICDCS 2015. Disco: a distributed localization scheme for mobile networks (IEEE, Columbus, 2015), pp. 527-536

14. J Cheng, Z Song, Q Ye, H Du, in 2016 IEEE International Conference on Communications, ICC 2016. Mil: a mobile indoor localization scheme based on matrix completion (IEEE, Kuala Lumpur, 2016), pp. 1-5

15. SA Kharidia, Q Ye, S Sampalli, J Cheng, H Du, L Wang, in 2014 10th International Conference on Mobile Ad-hoc and Sensor Networks, MSN 2014. Hill: A hybrid indoor localization scheme (IEEE, Maui, 2014), pp. 201-206

16. D Dardari, A Conti, J Lien, MZ Win, The effect of cooperation on uwb-based positioning systems using experimental data. EURASIP J. Adv. Sig. Process. 2008, 124-112411 (2008)

17. A Conti, D Dardari, MZ Win, in IEEE International Conference on Ultra-Wideband, ICUWB 2008. Experimental results on cooperative uwb based positioning systems (IEEE, Hannover, 2008)

18. K Liu, X Liu, X Li, in Proceeding of the 11th Annual International Conference on Mobile Systems, Applications, and Services. Guoguo: Enabling fine-grained indoor localization via smartphone (ACM, Taiwan, 2013), pp. 235-248

19. J Luo, HV Shukla, J-P Hubaux, in 25th IEEE International Conference on Computer Communications, INFOCOM 2016. Non-interactive location surveying for sensor networks with mobility-differentiated toa (IEEE, Barcelona, 2006), pp. 1241-1252

20. K Chintalapudi, A Padmanabha lyer, VN Padmanabhan, in Proceedings of the Sixteenth Annual International Conference on Mobile Computing and Networking. Indoor localization without the pain (ACM, Chicago, 2010), pp. 173-184

21. G Schroth, R Huitl, D Chen, M Abu-Alqumsan, A Al-Nuaimi, E Steinbach, Mobile visual location recognition. IEEE Signal Proc. Mag. 28(4), 77-89 (2011)

22. I Constandache, RR Choudhury, I Rhee, in The 29th Conference on Computer Communications, INFOCOM 2010. Towards mobile phone localization without war-driving (IEEE, San Diego, 2010), pp. 1-9

23. A LaMarca, Y Chawathe, S Consolvo, J Hightower, I Smith, J Scott, T Sohn, J Howard, J Hughes, F Potter, et al, in 3rd International Conference on Pervasive Computing. Place lab: Device positioning using radio beacons in the wild (Springer, Munich, 2005), pp. 116-133

24. PlaceEngine. http://www.placeengine.com. Accessed 26 Dec 2017

25. M Youssef, A Agrawala, in Proceedings of the 3rd International Conference on Mobile Systems, Applications, and Services. The horus wlan location determination system (ACM, Washington, 2005), pp. 205-218

26. Big Planet Tracks. https://code.google.com/archive/p/big-planet-tracks/. Accessed 26 Dec 2017

27. CWu, Z Yang, Y Zhao, Y Liu, in 32nd IEEE International Conference on Computer Communications, IEEE 2013. Footprints elicit the truth: Improving global positioning accuracy via local mobility (IEEE, Turin, 2013), pp. 490-494

28. CC Robusto, The cosine-haversine formula. Am Math. Mon. 64(1), 38-40 (1957)

29. T Cover, P Hart, Nearest neighbor pattern classification. IEEE Trans. Inf Theory. 13(1), 21-27 (1967) 